\title{
Movement parallax during locomotion
}

\author{
E. STURE ERIKSSON \\ University of Uppsala, S:t Larsgatan 2, S.752 20 Uppsala, Sweden
}

\begin{abstract}
A hy pothesis concerning the nature of movement parallax during locomotion is presented. According to the hypothesis, movement parallax constitutes a basic perceptual-motor mechanism able to produce veridical information as to spatial relations in three-dimensional space. Two experiments are reported verifying the hypothesis in that information due to movement parallax unequivocally dominated both the effects of proximal relative size and spatial anisotropy.
\end{abstract}

In view of the fact that even complex optical transformations are ambiguous with regard to absolute distance, it has been argued that space theory must necessarily incorporate nonvisual factors (Eriksson, 1973). In our laboratory, we have studied movement parallax as a basis for distance perception, ${ }^{1}$ and these and other studies (Ferris, 1972; Gogel \& Tietz, 1973) indicate that movement parallax constitutes a source of distance information but is not generally sufficient to produce veridical depth perception.

One of the most consistent complications has been the effect of relative stimulus-size implying a reversal of order in depth. Thus, the smaller of two proximal squares is perceived to be farther away than the larger, even if the smaller one actually is $2 \mathrm{~m}$ from the eye and the larger one is $4 \mathrm{~m}$. Neither prolonged observation nor a combination of lateral and vertical head movements is sufficient to prevent the nonveridical reversals in depth.

This result seems to indicate that movement parallax cannot be regarded as a basic principle in space perception. It is reasonable to expect, however, that veridical perception might be more likely to occur under conditions allowing a more natural interaction among the visual, proprioceptive, and vestibular systems. The purpose of the present investigation was to determine whether movement parallax will become more effective in a situation which permits the above-mentioned interaction.

\section{EXPERIMENT 1}

\section{Method}

A complex three-dimensional pattern of stimuli was arranged in a large dark room. S looked at this pattern when walking forward and backward in a path obliquely related to the stimulus pattern and where relative size during the whole path of locomotion was in conflict with parallax information. The arrangement of the experiment is shown in Fig. 1.

Stimuli. The stimuli consisted of 12 figures made by self-luminous homogeneous plastic and presented in a dark room with the walls covered by black cloth. The figures were placed in three planes perpendicular to a median plane through the zero point. In the farthest plane $(7 \mathrm{~m}$ from the zero point), there were four triangles, in the middle plane $(4.5 \mathrm{~m}$ from the zero point), four squares, and in the nearest plane $(2 \mathrm{~m}$ from the zero point), four circles. The triangles were $16.5 \mathrm{~cm}$ in size, the squares were $8.3 \mathrm{~cm}$, and the circles, $2.8 \mathrm{~cm}$. Thus, the triangles produced the largest proximal size and the circles the smallest.
The stimulus pattern viewed from a zero point $165 \mathrm{~cm}$ above the floor is shown in Fig. 2. Angular sizes and retinal disparities computed for positions in the median plane are presented in Table 1.

Observers. Six paid female Os participated in the experiment. The mean age was 18 years.

Procedure. $O$ was told to cover one eye (the nonpreferred one) with a black patch and was then led into the experimental room in darkness and positioned at the handrail. A black curtain occluded all the stimuli from view. $O$ was told to stand still (it was stressed that $O$ should refrain from making head

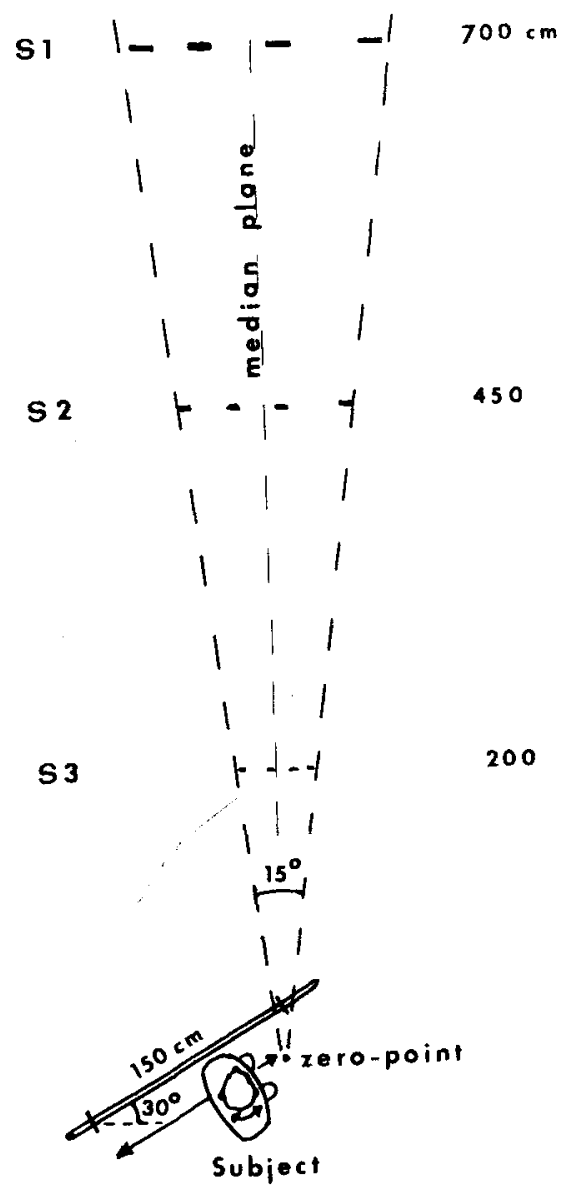

EXPERIMENTAL ARRANGEMENT

Fig. 1. Arrangement of Experiment I, top view. 
STIMULUS PATTERN

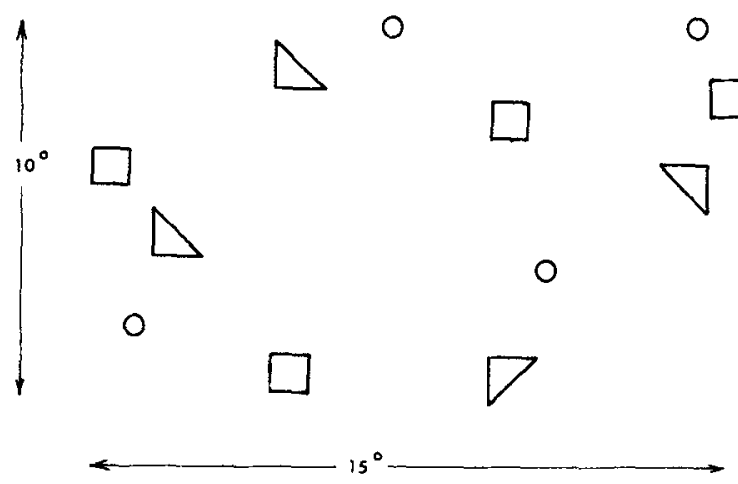

Fig. 2. Stimulus pattern in Experiment I viewed from the zero point.

Table 1

Sizes and Retinal Disparities for Stimuli Used in Experiment I

\begin{tabular}{lcccc} 
& \multicolumn{2}{c}{ Angular Sizes* } & & \\
\cline { 2 - 3 } $\begin{array}{l}\text { Stimulus } \\
\text { At Zero }\end{array}$ & $\begin{array}{c}\text { At End } \\
\text { Point }\end{array}$ & $\begin{array}{c}\text { Stimulus } \\
\text { Point }\end{array}$ & $\begin{array}{c}\text { Retinal } \\
\text { Pispar- } \\
\text { itiest }\end{array}$ \\
\hline $\begin{array}{l}\text { S3 } \\
\text { (Circles) }\end{array}$ & 0.80 & 0.53 & S1-S2 & 1.06 \\
$\begin{array}{l}\text { S2 } \\
\text { (Squares) }\end{array}$ & 1.06 & 0.88 & S2-S3 & 0.28 \\
$\begin{array}{l}\text { S1 } \\
\text { (Triangles) }\end{array}$ & 1.35 & 1.19 & S1-S3 & 1.34 \\
\hline
\end{tabular}

*Degrees of visual angle

tDegrees at zero point

movements) and to look to the left, whereupon the stimuli were presented. While standing still, $O$ was to judge whether the stimuli appeared in a plane or separated in depth (stating which figures were nearest to and farthest from the eye). Then the $O$ was told to walk forward and backward over the path of $150 \mathrm{~cm}$, noticing if any changes took place. Thereafter, the $O$ was asked to describe the order in depth, the distances to each plane in relation to the zero point, and the sizes of the figures. During these judgments, $O$ was told to walk continuously back and forth and to avoid fixating any particular figure. Finally, binocular vision under full-cue conditions in the lighted room was used, and $O$ was told to report whether any changes appeared. If changes appeared, new judgments were to be given. The reports were registered by a tape recorder.

\section{Results}

When $O$ was describing the static appearance, it became evident that the figures were generally perceived to be positioned in one plane some meters from the eye. These results agree with those obtained previously under similar conditions. However, as soon as $O$ began to move, the figures almost immediately separated into three distinct planes corresponding to the physical planes. The perceived order in depth was correct in $100 \%$ of the cases. Under the present conditions, it is therefore evident that movement parallax dominated the effects of relative size. The results with regard to judged distance are depicted in Fig. 3 ( $\mathrm{SD}=55,155$, and $258 \mathrm{~cm}$ for circles, squares, and triangles, respectively). According to Fig. 3, judged distance (J) is a linear function of physical distance (S), i.e., $J=.78 \mathrm{~S}$. Using the distance estimates from the full-cue situation as criterion, it is possible to calibrate judged distance to obtain an estimate of perceived distance (Gogel, 1969). The correction coefficient is 1.11 , which yields $\mathrm{P}=$ $.86 \mathrm{~S}$. Thus, perceived distance $(\mathrm{P})$ is proportional to physical distance. As shown in Fig. 3, there exists a slight underestimation of perceived distance, an outcome which is consistent with earlier findings in the study of distance perception (Gogel, 1968, 1960).

\section{EXPERIMENT II}

Spatial anisotropy implies that the upper parts of a pattern tend to be perceived as farther away than the lower parts, irrespective of the true positions. This effect has been obtained in our previous experiments with lateral head movements when the stimuli were placed in a vertical or oblique row. In Experiment II, it was asked whether the same outcome would be obtained when $O$ moved in natural locomotion. In the present situation, the information due to movement parallax was counteracted both by relative size and anisotropy. The conditions therefore constitute a stronger test of the functional effectiveness of movement parallax during locomotion.

\section{Method}

Stimuli. The stimuli consisted of five self-luminous squares made of homogeneous plastic. They were placed 3, 4, 5, 6, and $7 \mathrm{~m}$ from the starting point (zero point). The sizes were $3,5.2$, $8.4,12$, and $16.3 \mathrm{~cm}$, respectively. The nearest (smallest) square was placed $183 \mathrm{~cm}$ and the farthest (largest) one $100 \mathrm{~cm}$ above the floor. The arrangement of the experiment is shown in Fig. 4, and the stimulus pattern is depicted in Fig. 5. Angular sizes,

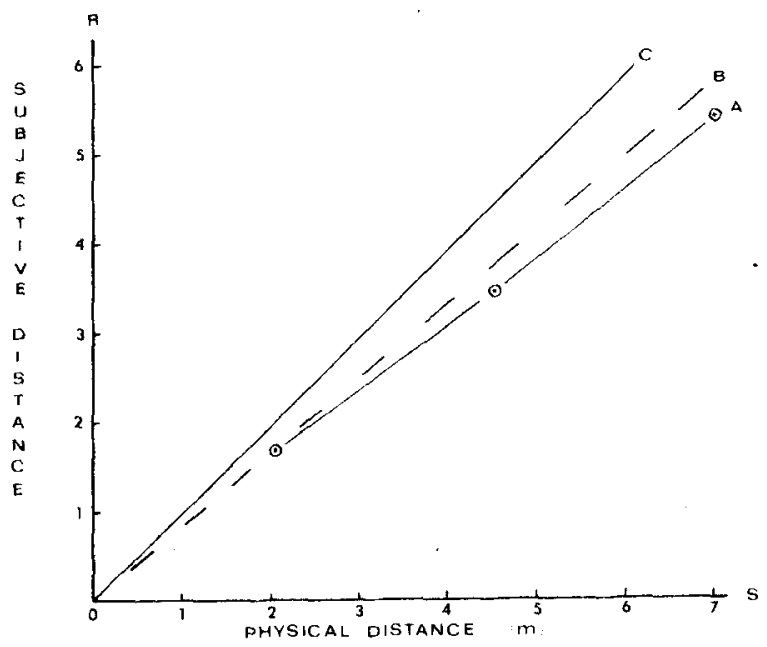

Fig. 3. Results of Experiment I. Curve A shows judged distance as a function of physical distance, Curve B depicts perceived distance as a function of physical distance after calibration. Curve $\mathrm{C}$ shows veridicality. 
separations, and retinal disparities for the stimuli computed for a zero point $165 \mathrm{~cm}$ above the floor are presented in Table 2. The room was kept dark during the experiment, and pieces of black cloth were placed in such a way as to prevent reflections from ceiling, floor, or walls.

Procedure. $O$ was led into the room in darkness and positioned at the handrail. Monocular vision with preferred eye was $u$ sed. The curtain was pulled away and $O$ was told to state whether the upper or the lower square was nearest to the eye during monocular static conditions. Then $\mathrm{O}$ was asked to move $1 \mathrm{~m}$ forward and then back up to the starting point while estimating the two distances with regard to the starting point. Then $O$ was told to move $2 \mathrm{~m}$ forward, and then to back up again while viewing the stimuli and reporting if any distance changes had taken place. During this trial, the sizes of the largest and the smallest squares were also to be judged. Finally, the room was lighted, and $O$ estimated the distances during binocular full-cue conditions. Eight female Os were used in the experiment.

\section{Results}

When the Os were standing at the starting point, the upper (nearest) square was usually perceived as the farthest one. In one case, the pattern appeared as two-dimensional. No O perceived the upper square as the nearest one. However, during locomotion, all the Os perceived a clear reversal to the veridical order in depth.

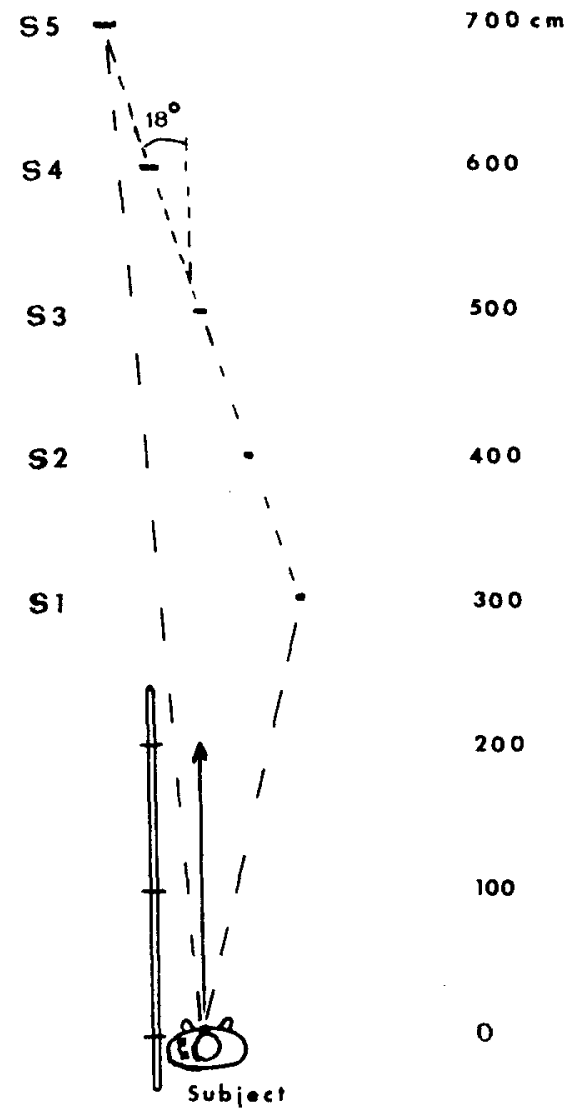

ARR A NGEMENT

Fig. 4. Arrangement of Experiment II, top view.
STIMULUS PATTERN

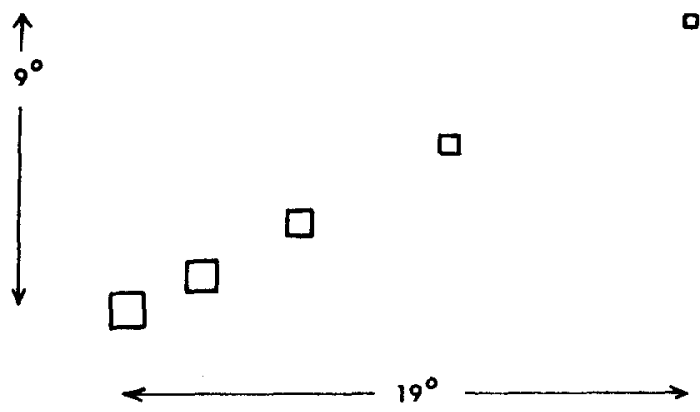

Fig. 5. Stimulus pattern in Experiment II viewed from the zero point.

Table 2

Angular Sizes, Separations, and Retinal Disparities for Stimuli Used in Experiment II

\begin{tabular}{|c|c|c|c|c|c|}
\hline \multirow{2}{*}{ Stimulus } & \multicolumn{2}{|c|}{ Angular Sizes* at } & & & \\
\hline & Zero & Point & & & \\
\hline S1 & $0<4$ & 141 & & & \\
\hline S2 & 0.74 & 1.46 & & & \\
\hline S3 & 0.94 & 1.61 & & & \\
\hline S4 & 1.15 & 1.72 & & & \\
\hline S5 & 1.33 & 1.87 & & & \\
\hline \multirow{3}{*}{$\begin{array}{c}\text { Stimulus } \\
\text { Pair }\end{array}$} & \multicolumn{2}{|c|}{$\begin{array}{c}\text { Vertical } \\
\text { Separation* at }\end{array}$} & \multicolumn{2}{|c|}{$\begin{array}{c}\text { Horizontal } \\
\text { Separation* at }\end{array}$} & \multirow{3}{*}{$\begin{array}{c}\text { Dis- } \\
\text { parity } \dagger\end{array}$} \\
\hline & Zero & Point & Zero & Point & \\
\hline & Point & 200 & Point & 200 & \\
\hline S1-S2 & 3.8 & 10.6 & 8.7 & 24.4 & 0.31 \\
\hline S2-S3 & 2.4 & 4.2 & 4.8 & 9.7 & 0.19 \\
\hline S3-S4 & 1.4 & 2.0 & 3.1 & 4.7 & 0.12 \\
\hline S4-S5 & 1.1 & 0.9 & 2.2 & 2.2 & 0.09 \\
\hline
\end{tabular}

In four cases, the reversal occurred during the walk toward Point $200 \mathrm{~cm}$ and in four cases during locomotion toward Point $100 \mathrm{~cm}$. The reversal was followed by spontaneous remarks indicating perceptual changes in the direction of veridicality. The nearest (upper) square was judged to be $242 \mathrm{~cm}(S D=36 \mathrm{~cm})$ from the starting point, and the farthest (lowest) square was judged to be $458 \mathrm{~cm}(\mathrm{SD}=86 \mathrm{~cm})$ from that point. These values are very close to the distance function depicted in Fig. 3. It was found that judged distance could be described by the function $J=.70 \mathrm{~S}$. Since the correction coefficient obtained in the full-cue condition was 1.25 , we obtain $\mathrm{P}=.88 \mathrm{~S}$ for perceived distance as a function of physical distance.

On the basis of this experiment, it is concluded that information due to movement parallax during locomotion unequivocally dominates the joint effects of proximal relative size and spatial anisotropy.

\section{DISCUSSION}

The results of the experiments provide an 
unambiguous answer to the experimental questions raised in this paper. Movement parallax during locomotion is an effective source of veridical space perception. Under our conditions, it has been established that movement parallax can dominate the effects of both relative size and anisotropy. This result may have far-reaching theoretical consequences, since it implies that movement parallax may constitute the primary perceptual-motor mechanism which (a) differentiates objects in perceptual space, (b) produces a calibrated egocentric scale with regard to object distances, and (c) produces information concerning one's own movements in relation to physical objects.

The above interpretation, however, is restricted to situations characterized by stimulus complexity. As indicated in the introduction, less complex stimuli may produce quite different results, although from a geometrical point of view the same principle is involved in both cases. For the present, it is not clear why relative size and even spatial anisotropy should dominate parallax information under less complex conditions. Since the body-state information (proprioception including kinesthetic and vestibular information) reasonably must be the same during locomotion irrespective of the optical input, the explanation apparently has to be found in connection with one of two hypothetical perceptual functions, which in the following are termed the optical hypothesis and the interaction hypothesis.

According to the optical hypothesis, the reversals in depth are due to reduced and ineffective optical information. In order to produce the correct order in depth, it would be sufficient to use a stationary $O$ and complex optical changes. Even if this hypothesis turns out to be true, it is, nevertheless, insufficient as an explanation of the most important aspect of the perceptions, namely, the veridical distances, because even complex optical changes are ambiguous with regard to absolute distances (Eriksson, 1973).

According to the interaction hypothesis, it is required that the $O$ be active in the sense that he executes head movements or is under locomotion. The interaction hypothesis is characterized by two aspects. With regard to reversals in depth, it implies that a certain optical transformation obtained under locomotion will produce a greater amount of correct orders (i.e., fewer reversals) than will be obtained with a stationary $O$ and the identical optical transformation pattern. In order to test the optical vs the interaction hypothesis in this context, a new experimental paradigm is required. According to this approach, the perceptual effects should be studied when a group of active Os view a certain optical pattern compared with another group where the same optical pattern (changing identically) is presented to stationary Os. In this way, it should be possible to define the critical factors and to decide whether they are optical-proprioceptive or if they are purely optical.

With regard to absolute distances, the interaction hypothesis implies that veridicality of distances for unfamiliar objects cannot be obtained unless there is an interaction between body-state information and optical information. It is conceivable that to be effective, the interaction must be based on a certain complexity (optical or optical-proprioceptive, or both). Accordingly, a decreased complexity will be followed by perceptual instability and increasing dominance of secondary influences such as relative size, anisotropy, and the like, as some of our previous experiments have demonstrated. Therefore, future experiments should try to clarify the dependence of perceived distance on the above-mentioned interaction, i.e., on different optical factors and on locomotion factors such as path length of locomotion, curvilinear vs straight paths, different kinds of head movements, and interactions between head movements and locomotions.

\section{REFERENCES}

Eriksson, E. S. Distance perception and the ambiguity of visual stimulation: A theoretical note. Perception \& Psychophysics, $1973,13,379-381$.

Ferris, $\dot{S}$. H. Motion parallax and absolute distance. Journal of Experim ental Psy chology, 1972, 95, 258-263.

Gogel, W. C. The perception of shape from binocular disparity cues. Journal of Psychology, 1960, 5, 179-192.

Gogel, W. C. The effect of set on perceived egocentric distance. Acta Psychologica, 1968, 28, 283-292.

Gogel. W. C. The sensing of retinal size. Vision Research, 1969, 9, 1079-1094.

Gogel, W. C., \& Tietz, J. D. Absolute motion parallax and the specific distance tendency. Perception \& Psychophysics, 1973, 13, 28 4-292.

\section{NOTE}

1. Laboratory reports describing this work are available from the author upon request.

(Received for publication November 9, 1973; revision accepted March 11, 1974.) 